\title{
Association between postoperative changes in the gut microbiota and pseudopsia after cardiac surgery: prospective observational study
}

\author{
Masaki Maekawa ${ }^{1}$, Kenji Yoshitani ${ }^{1 *} \mathbb{C}$, Musashi Yahagi ${ }^{1}$, Takashi Asahara ${ }^{2}$, Yoshiyuki Shishido $^{2}$, \\ Satsuki Fukushima ${ }^{3}$, Naoki Tadokoro ${ }^{3}$, Tomoyuki Fujita ${ }^{3}$ and Yoshihiko Ohnishi ${ }^{1}$
}

\begin{abstract}
Background: Delirium after cardiac surgery affects mortality, but the mechanism remains unclear. Previous studies have reported gut microbiota are associated with brain activity. Systemic inflammation and antibiotics can damage the gut microbiota after cardiac surgery. We aimed to investigate changes in the gut microbiota and the association between the gut microbiota and delirium after cardiac surgery.

Methods: Twenty-one patients who underwent cardiac surgery were enrolled. Microbiota counts and fecal organic acid concentrations were measured in fecal samples harvested before surgery, just after surgery, and before discharge. To quantify the microbiota, we extracted total RNA fractions and examined gut microbiota composition using $16 \mathrm{~S}$ and $23 \mathrm{~S}$ rRNA-targeted quantitative-reverse Transcription-PCR. Postoperative delirium, insomnia, and pseudopsia were assessed for 1 week. Postoperative total bacterial counts changed significantly from $10.2 \pm 0.2 \log _{10}$ cells $/ \mathrm{g}$ of feces to $9.8 \pm 0.5$ in the first postoperative samples $(p=0.003)$ and $10.0 \pm 0.4$ in the samples before discharge $(p=0.039)$. Fecal pH was $6.9 \pm 0.6$ before surgery and $7.4 \pm 0.7$ in the first postoperative samples $(p=0.001)$. Postoperative Staphylococcus and Pseudomonas counts were significantly higher in patients with postoperative pseudopsia than in patients without pseudopsia ( $3.2 \pm 1.3$ vs. $5.4 \pm 0.9 ; p=0.012$ and $1.7 \pm 0.8$ vs. $4.6 \pm 2.7 ; p=0.001$ ).

Conclusions: Total bacterial counts were significantly lower after surgery and until discharge. Fecal pH was significantly higher than preoperative levels. Staphylococcus and Pseudomonas counts were significantly higher in patients with postoperative pseudopsia.
\end{abstract}

Keywords: Delirium, Microbiota, Gut-brain axis, Cardiac surgery

\section{Background}

Postoperative delirium after cardiac surgery is a serious complication and an independent predictor of worse prognosis [1]. Some studies have reported risk factors for delirium, such as age and inflammation $[2,3]$.

\footnotetext{
*Correspondence: ykenji@ncvc.go.jp

${ }^{1}$ Department of Anesthesiology, National Cerebral and Cardiovascular Center, Suita, Japan

Full list of author information is available at the end of the article
}

However, details about postoperative delirium remain to be clarified.

Previous studies have demonstrated that individual differences in the gut microbiota influence health status $[4$, 5] as well as brain activity, which is referred to as microbiota-gut-brain communication [6, 7]. Patients who undergo cardiac surgery with cardiopulmonary bypass who develop systemic inflammation [8] and receive multiple drugs experience damage to their gut flora $[9,10]$. Some studies claim that damaged gut flora may lead to 
perioperative complications [11]. We hypothesize that postoperative delirium can be associated with damage to gut flora.

This single-center prospective observational study aimed to identify changes in gut flora after cardiopulmonary bypass and differences in gut flora between patients with and without postoperative pseudopsia or insomnia, which are symptoms of postoperative delirium.

\section{Methods}

\section{Study design}

This study was a single-center prospective observational study to investigate perioperative changes in the gut microbiota associated with cardiac surgery and the association between gut flora and postoperative pseudopsia or insomnia. The study was approved by the institutional ethics committee and conforms to the ethical norms and standards in the Declaration of Helsinki.

\section{Patients}

In this study, 21 adult patients who underwent elective cardiac surgery with cardiopulmonary bypass were enrolled. Participants provided written informed consent. Exclusion criteria included another major illness except for preoperative hypertension, diabetes mellitus, or dyslipidemia; selective cerebral perfusion; deep hypothermic circulatory arrest; aortic surgery; and preoperative administration of antibiotics. Preoperative and perioperative patient characteristics were collected from medical records.

\section{Delirium assessment}

Postoperative delirium was assessed using the CAM-ICU scale (https://www.icudelirium.org/medical-profession als/delirium/monitoring-delirium-in-the-icu) during the week after extubation, which has been used most frequently $[12,13]$. Furthermore, postoperative pseudopsia and insomnia were investigated simultaneously. They are not included in the CAM-ICU, but are included in the Diagnostic and Statistical Manual of Mental Disorders, 5th edition. Symptoms of delirium were also included. Pseudopsia and insomnia were assessed by Intensive Care Unit (ICU) physicians. The insomnia group included patients who had insomnia for more than 2 days after surgery to exclude the effects of general anesthesia. The pseudopsia group contained patients who had pseudopsia on more than one occasion.

\section{Anesthetic management}

All patients underwent induction of general anesthesia with $0.5-1 \mathrm{mg} / \mathrm{kg}$ of midazolam, $2-10 \mu \mathrm{g} / \mathrm{kg}$ of fentanyl, and $0.6-1 \mathrm{mg} / \mathrm{kg}$ of rocuronium. Anesthesia was maintained with $4-6 \mathrm{mg} / \mathrm{kg} / \mathrm{h}$ of propofol, $30 \mu \mathrm{g} / \mathrm{kg} / \mathrm{h}$ of remifentanil, and $0.4-0.5 \mathrm{mg} / \mathrm{kg} / \mathrm{h}$ of rocuronium. Sevoflurane was sometimes added in order to control blood pressure for short periods of time. Local anesthesia was not always used. All patients received $3 \mathrm{~g} /$ day of cefazolin on postoperative day (POD) 0 and 1 .

\section{Fecal sample collection}

Patients collected their own fecal samples at three time points. The first sample, which served as the control, was harvested a few days before surgery. The second sample was from the first or second bowel movement after surgery. In general, the first postoperative sample was harvested but some patients were critically ill and the second sample was harvested. The last sample was harvested sometime between (POD) 6 and 8. Patients placed the fecal samples directly into two tubes (approximately $1.0 \mathrm{~g} /$ tube). One tube contained $2 \mathrm{~mL}$ RNAlater (an RNA stabilization solution; Ambion, Austin, TX, USA), and the other was empty. The samples with RNAlater were placed in a refrigerator at $4{ }^{\circ} \mathrm{C}$ for analysis of the fecal microbiota. The other samples were placed in a freezer at $-80{ }^{\circ} \mathrm{C}$ within $30 \mathrm{~min}$ of excretion for analysis of fecal organic acid concentrations and $\mathrm{pH}$. Samples were transported to the Yakult Central Institute at $-20^{\circ} \mathrm{C}$ for analysis. The patient's identity, clinical information, and study group were unknown to the technicians performing the analysis.

\section{Gut microbiota analysis}

To quantify the bacteria present in the samples, we extracted total RNA fractions from the fecal samples using previously described methods [14-17]. We examined gut microbiota composition using $16 \mathrm{~S}$ and $23 \mathrm{~S}$ rRNA targeted quantitative Reverse Transcription PCR (qRT-PCR) using the Yakult Intestinal Flora-SCAN analysis system (YIF-SCAN ${ }^{\circledR}$; Yakult Honsha, Tokyo, Japan) [16]. Three serial dilutions of each extracted RNA sample were used for rRNA-targeted qRT-PCR. Threshold cycle values in the linear range of the assay were applied to the standard curve to obtain the corresponding bacterial cell count for each fecal or blood sample. In this study, predominant anaerobes present in the human intestine (Clostridium coccoides group, Clostridium leptum subgroup, Bacteroides fragilis group, Bifidobacterium, Atopobium cluster, and Prevotella) and intestinal subdominant populations (Clostridium difficile, Clostridium perfringens, Lactobacillus, Enterobacteriaceae, Enterococcus, Streptococcus, Staphylococcus, and Pseudomonas) were examined. The specificity of the qRT-PCR assay using group-specific, genus-specific, and species-specific primers was determined as described previously [14, 15, $17-19]$. 


\section{Short-chain fatty acids concentration (SCFAs) and pH measurement}

Concentrations of fecal SCFAs were measured as described previously [20] with slight modifications. Briefly, the frozen samples were homogenized in fourfold volumes of $0.15 \mathrm{~mol} / \mathrm{l}$ perchloric acid and allowed to stand at $4{ }^{\circ} \mathrm{C}$ for $12 \mathrm{~h}$. The suspension was subjected to centrifugation at $20,400 \times g$ at $4{ }^{\circ} \mathrm{C}$ for $10 \mathrm{~min}$. The resultant supernatant was passed through a filter with a pore size of $0.45 \mu \mathrm{m}$ (Millipore Japan, Tokyo, Japan). The sample was analyzed for organic acids using a highperformance liquid chromatography system (Waters 432 Conductivity Detector, Waters, Milford, MA, USA). Organic acid concentrations were calculated with the use of external standards and expressed as $\mu \mathrm{mol} / \mathrm{g}$ of wet feces. The lower limits for fecal organic acid concentrations using this procedure were $0.075 \mu \mathrm{mol} / \mathrm{g}$ for succinic acid, $0.2 \mu \mathrm{mol} / \mathrm{g}$ for lactic acid, $0.05 \mu \mathrm{mol} / \mathrm{g}$ for formic acid, $0.4 \mu \mathrm{mol} / \mathrm{g}$ for acetic acid, $0.5 \mu \mathrm{mol} / \mathrm{g}$ for propionic acid, $0.55 \mu \mathrm{mol} / \mathrm{g}$ for butyric acid, $0.8 \mu \mathrm{mol} / \mathrm{g}$ for isovaleric acid, and $0.65 \mu \mathrm{mol} / \mathrm{g}$ for valeric acid. Fecal $\mathrm{pH}$ was measured by directly inserting the glass electrode of a D-51 pH meter (Horiba Seisakusho, Tokyo, Japan) into a sample of homogenized feces.

\section{Statistical analysis}

Postoperative microbiota counts and fecal organic acid concentrations were compared with preoperative control values using the paired t test. If microbiota counts were lower than the limit of detection, the count of the sample was treated as half of the limit of detection value. Moreover, microbiota counts and fecal organic acid concentrations were compared between patients with or without pseudopsia and insomnia using the unpaired test. Analyses were performed using Stata/SE, version 16 (StataCorp, College Station, TX, USA).

\section{Results}

The perioperative characteristics parameters of the 21 patients are shown in Table 1. There were no patients with delirium diagnosed based on the CAMICU. Three patients had pseudopsia and 11 patients had insomnia for more than 2 days. Consequently, we could not compare differences between patients with and without delirium diagnosed based on the CAMICU. No unexpected critical adverse events were observed perioperatively. All patients were extubated in the ICU; 20 of 21 patients were extubated on the day of surgery. Only one patient remained intubated until the next morning. All patients started oral intake and physical rehabilitation on POD 1. Cefazolin was used in 19 patients. Two patients received additional
Table 1 Perioperative characteristics and parameters

\begin{tabular}{ll}
\hline & $\begin{array}{l}\text { Number } \\
\text { of patients (\%) } \\
\text { (n=21) }\end{array}$ \\
\hline Age (years), median (range) & $62(22-80)$ \\
Male sex, n (\%) & $16(76.2)$ \\
Smoking status & \\
Never smoker & $9(42.9)$ \\
Previous smoker & $10(47.6)$ \\
Current smoker & $2(9.5)$ \\
Hypertension & $11(52.4)$ \\
Diabetes mellitus & $3(14.3)$ \\
Dyslipidemia & $9(42.9)$ \\
Operation, n (\%) & \\
Valve surgery & $17(81.0)$ \\
Valve surgery+ CABG & $2(9.5)$ \\
Tumor removal & $1(4.8)$ \\
Myectomy & $1(4.8)$ \\
Dairy-based ingestion of lactobacillus, n (\%) & $10(47.6)$ \\
Postoperative delirium, n (\%) & $0(0)$ \\
Postoperative pseudopsia, n (\%) & $3(14.3)$ \\
\hline Valverative insomnia, n (\%) & $11(52.4)$ \\
\hline
\end{tabular}

Valve surgery included aortic valve replacement $(n=9)$, mitral valve repair $(\mathrm{n}=8)$, and mitral valve replacement $(\mathrm{n}=2)$

$C A B G$ coronary artery bypass grafting

antibiotics because of suspected postoperative infection. In one patient, doripenem hydrate was added on POD 3. In another patient, vancomycin and meropenem hydrate were added on POD 6.

On average, the first postoperative samples were harvested on POD 3.2. More specifically, samples were harvested on POD 2 in 3 patients, POD 3 in 12 patients, POD 4 in 4 patients, and POD 6 in 1 patient. One patient could not harvest the second sample (first postoperative sample). All analyses comparing preoperative versus first postoperative samples were based on the other 20 patients.

\section{Total microbiota counts and concentrations of SFCAs in feces}

All values were compared with preoperative levels. Microbiota counts are presented as $\log _{10}$ cells/g of feces. Figure 1 shows the perioperative changes in microbiota count. Compared with preoperative total microbiota counts $(10.2 \pm 0.2)$, counts in the first and second postoperative fecal samples were significantly lower $(9.8 \pm 0.5$; $p=0.003$ and $10.0 \pm 0.4 ; p=0.039$, respectively).

Among obligate anaerobes, Clostridium coccoides group counts were significantly lower in the first postoperative fecal samples than in preoperative samples 


\section{Perioperative change in microbiota count}

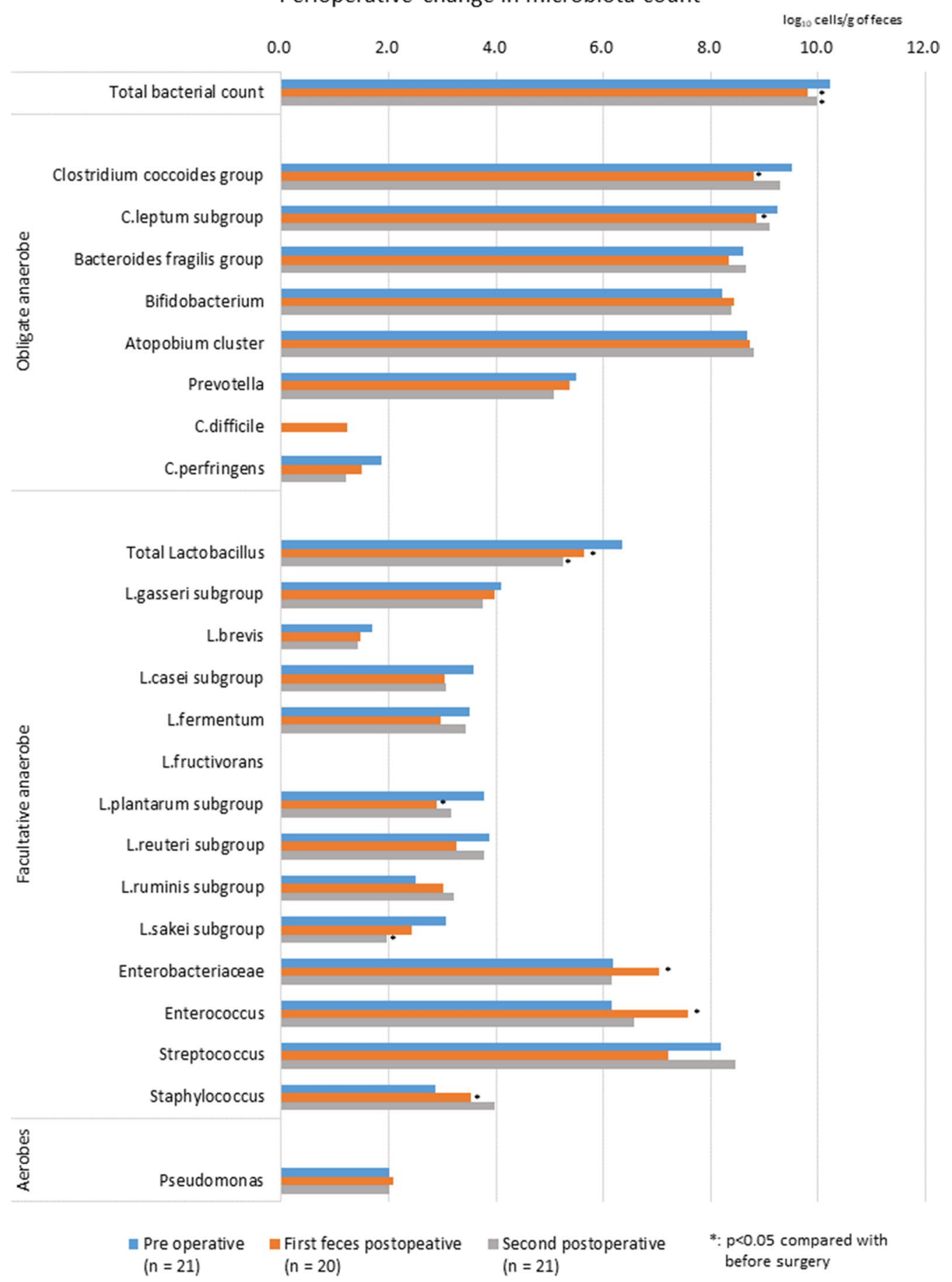

Fig. 1 Perioperative changes in microbiota counts. Blue bars represent preoperative counts, orange bars represent first postoperative counts, and gray bars represent second postoperative counts (around POD 7). Each postoperative microbiota count was compared with the corresponding preoperative count with the paired t test. Asterisk shows significant differences. POD postoperative day

$(8.8 \pm 0.7$ vs. $9.5 \pm 0.4 ; p=0.001)$. Clostridium leptum subgroup counts were also lower in the first postoperative samples $(8.9 \pm 0.8$ vs. $9.2 \pm 0.8 ; p=0.040)$.
Among facultative anaerobes, postoperative total Lactobacillus counts, both in the first postoperative and second postoperative samples (collected around POD 7 ), were significantly lower: preoperative; $6.4 \pm 1.4$; 
first postoperative, $5.6 \pm 1.4(p=0.013)$; and second postoperative, $5.3 \pm 2.4(p=0.025)$. Of note, L. plantarum subgroup counts were lower in the first postoperative fecal samples: preoperative, $3.8 \pm 1.6$ versus first postoperative, $2.9 \pm 1.3(p=0.038)$. L. sakei subgroup counts were lower in the second postoperative samples collected around POD 7: preoperative, $3.1 \pm 2.1$ versus second postoperative, $2.0 \pm 1.3(p=0.036)$.

By contrast, Enterobacteriaceae counts were significantly higher in the first postoperative fecal samples compared with preoperative samples (7.0 \pm 0.7 vs. $6.2 \pm 1.5, p=0.031)$. Enterococcus counts were also significantly higher in the first postoperative fecal samples compared with preoperative samples $(7.6 \pm 2.0$ vs. $6.2 \pm 1.7 ; p=0.009)$. Staphylococcus counts were significantly higher in the second postoperative fecal samples compared with preoperative samples $(4.0 \pm 1.2$ vs. $2.9 \pm 1.5 ; p=0.002$ ).

Figure 2 shows perioperative changes in fecal SFCAs concentrations. The first postoperative and second postoperative fecal samples were significantly more alkalinized than preoperative samples: preoperative $\mathrm{pH}$, $6.9 \pm 0.6$; first postoperative $\mathrm{pH}, 7.4 \pm 0.7(p=0.011)$; and second postoperative $\mathrm{pH}, 7.4 \pm 0.7(p=0.001)$. Postoperative butyric acid concentrations were lower after surgery: preoperative, $8.7 \pm 5.9 \mu \mathrm{mol} / \mathrm{g}$ of feces; first postoperative, $4.9 \pm 3.1 \mu \mathrm{mol} / \mathrm{g}(p=0.026)$; second preoperative, $5.2 \pm 5.5 \mu \mathrm{mol} / \mathrm{g}(p=0.008)$.

\section{Microbiota counts and concentrations of fecal SFCAs in patients with and without pseudopsia}

Microbiota counts and fecal SFCAs concentrations of the pseudopsia group were compared with those of the

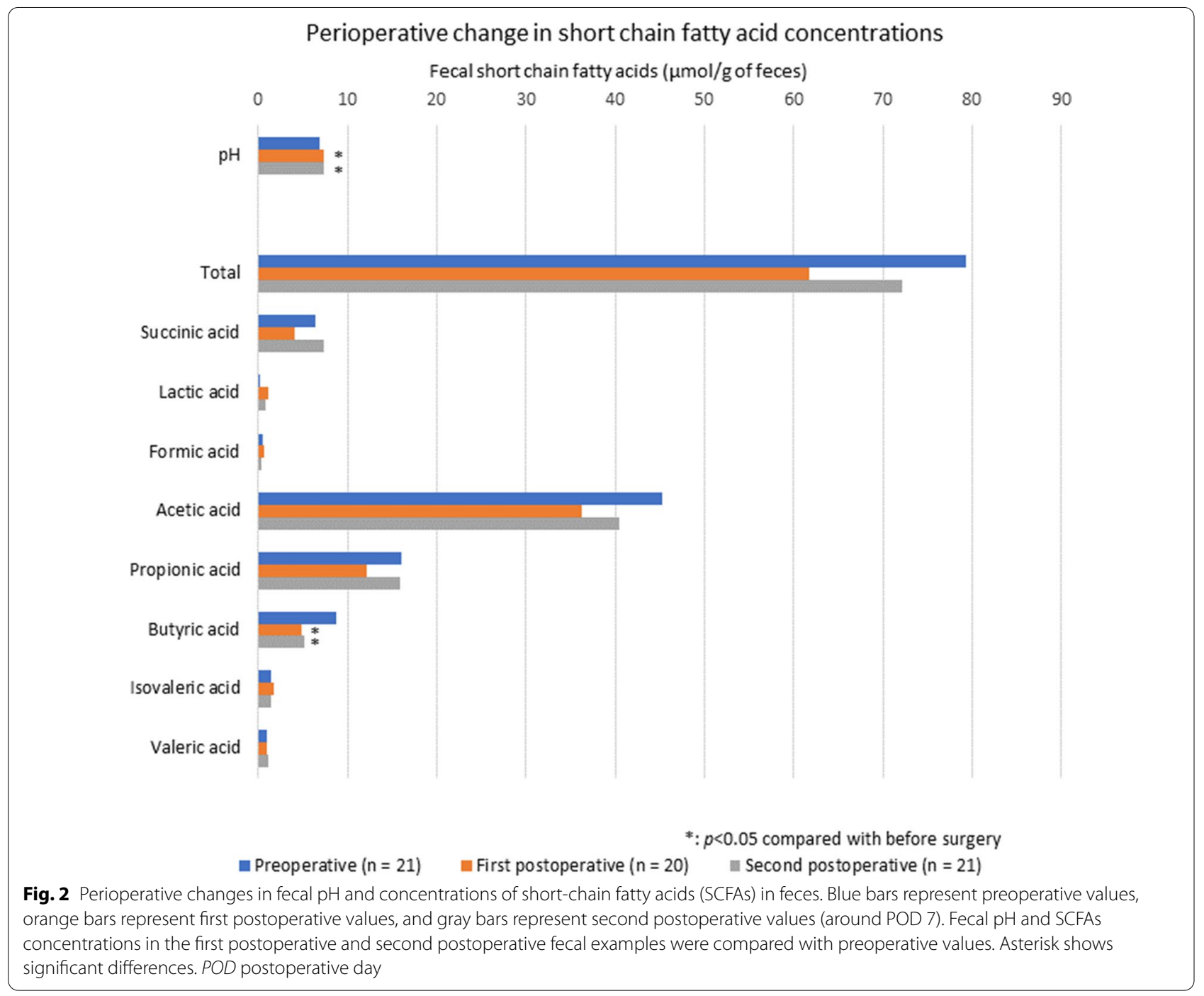




\section{Microbiota count on patients with versus without pseudopsia}

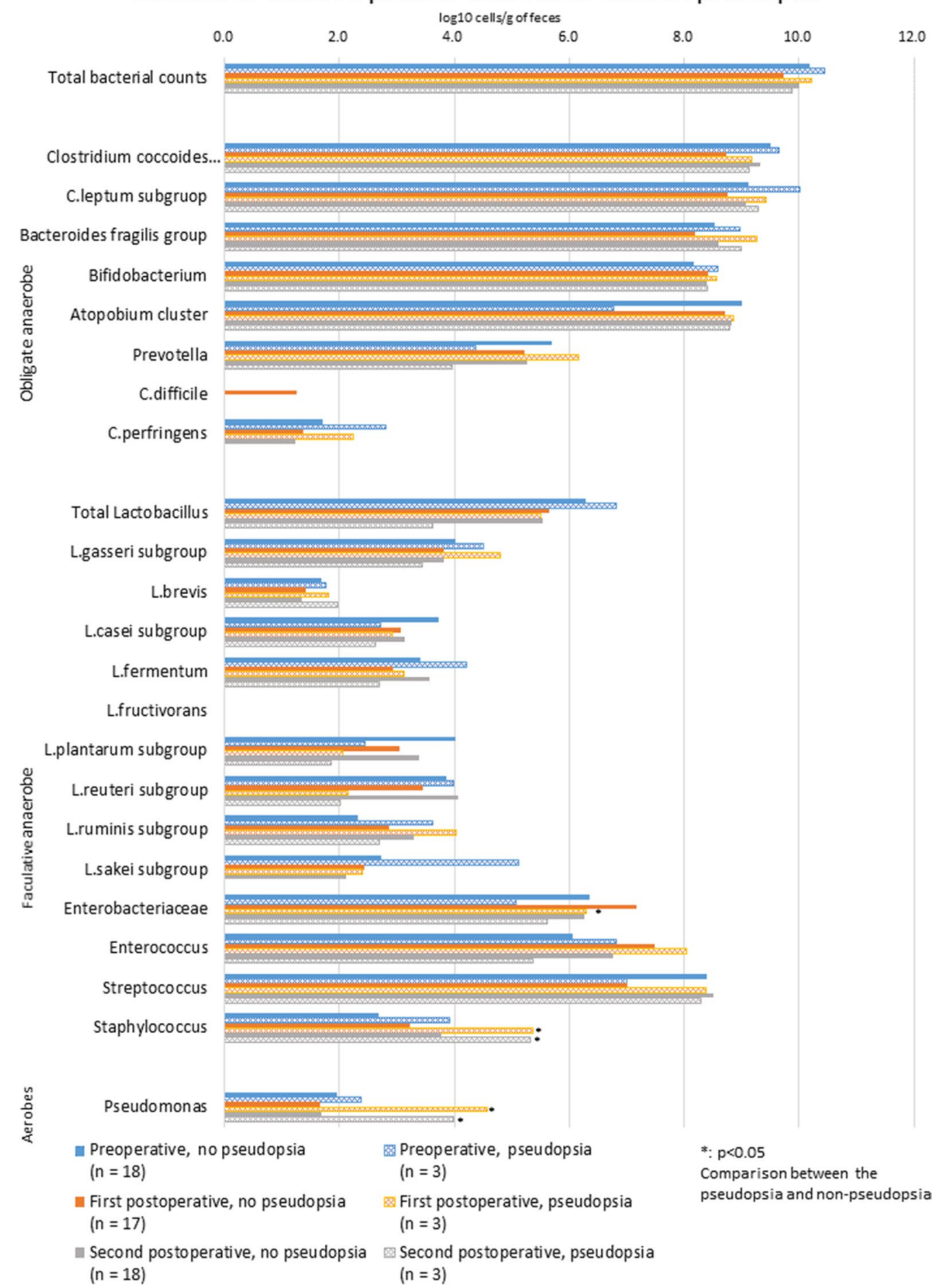

Fig. 3 Perioperative changes in microbiota counts in patients with versus without pseudopsia. Blue bars represent preoperative counts, orange bars represent first postoperative counts, and gray bars represent second postoperative counts (around POD 7). The mesh pattern depicts the pseudopsia group. For each measurement timepoint, differences between patients with and without pseudopsia were compared using the $t$ test. Asterisk shows significant differences. $P O D$ postoperative day 


\section{Short chain fatty acid concentrations in patients with and without pseudopsia} Fecal short-term fatty acids ( $\mu \mathrm{mol} / \mathrm{g}$ of feces)

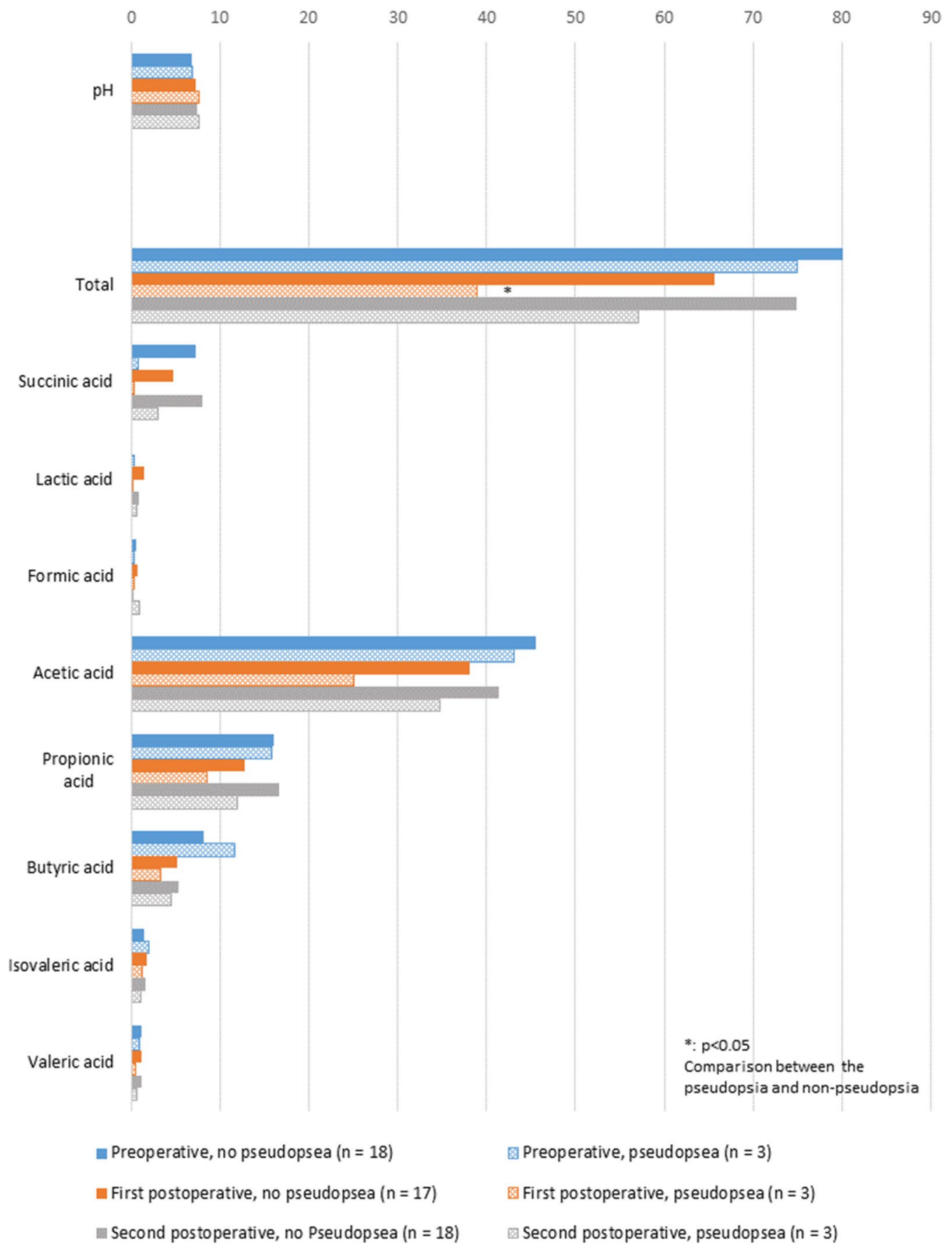

Fig. 4 Perioperative changes in fecal pH and concentrations of short-chain fatty acids in feces between patients with and without pseudopsia. Blue bars represent preoperative values, orange bars represent first postoperative values, and gray bars represent second postoperative values (around POD 7). The mesh pattern depicts the pseudopsia group. For each measurement timepoint, differences between patients with and without pseudopsia were compared using the t test. Asterisk shows significant differences. POD postoperative day 
non-pseudopsia group (Figs. 3, 4). Postoperative Staphylococcus counts in the pseudopsia group were significantly higher than in the non-pseudopsia group for first postoperative fecal samples $(3.2 \pm 1.3$ vs. $5.4 \pm 0.9$; $p=0.012$ ). Similarly second postoperative fecal samples had higher Staphylococcus counts than in the non-pseudopsia group (5.3 \pm 0.4 vs. $3.8 \pm 1.1 ; p=0.034)$. Postoperative Pseudomonas counts were significantly higher in the pseudopsia group than in the non-pseudopsia group for first postoperative fecal samples $(4.0 \pm 2.3$ vs. $1.7 \pm 0.7 ; p=0.001)$ and second postoperative fecal samples $(4.6 \pm 2.7$ vs. $1.7 \pm 0.8 ; p=0.001)$. Postoperative Enterobacteriaceae counts in first postoperative fecal samples were higher in the pseudopsia group than in the non-pseudopsia group ( $6.3 \pm 0.7$ vs. $7.2 \pm 0.6 ; p=0.001)$. Atopobium cluster counts were only significantly different across groups for preoperative fecal samples. Preoperative Atopobium cluster counts of the pseudopsia group were significantly lower than those of the nonpseudopsia group (6.8 \pm 3.8 vs. $9.0 \pm 0.7 ; p=0.001)$. First postoperative fecal sample concentrations of total fecal SFCAs in the pseudopsia group were significantly lower than in the non-pseudopsia group $(38.9 \pm 8.5$ vs. $65.7 \pm 17.1 \mu \mathrm{mol} / \mathrm{g}$ of feces; $p=0.018$ ).

\section{Microbiota counts and concentrations of fecal SFCAs in patients with and without insomnia}

In preoperative fecal samples, there were significant differences in counts of Enterobacteriaceae (insomnia group, $5.5 \pm 1.9 \log _{10}$ cells/g of feces; non-insomnia group, $6.9 \pm 0.5 \log _{10}$ cells/g of feces; $p=0.044$ ) and Enterococcus (insomnia group: $5.5 \pm 1.8$; non-insomnia group, $6.9 \pm 1.4 ; p=0.049$ ). However, there were no significant postoperative differences. There were also no significant differences in concentrations of fecal SFCAs in patients with versus without insomnia.

\section{Discussion}

This study revealed changes in the gut microbiota after cardiac surgery that persisted for 1 week even when patients restarted oral intake just after surgery. Total bacterial counts were significantly lower in the first and second postoperative samples, as well as both obligate anaerobe and facultative anaerobe counts. Fecal pH was significantly higher in the first and second postoperative fecal samples. In patients with pseudopsia, the first and second postoperative fecal samples had significantly higher Staphylococcus and Pseudomonas counts. Total fecal SFCAs concentrations were significantly lower in the first postoperative fecal samples.

In a previous study, obligatory anaerobe counts in patients with systemic inflammatory response syndrome were significantly lower than in normal subjects. Fecal
$\mathrm{pH}$ was higher and fecal organic acid concentrations were dramatically lower in such patients [10]. In this study, similar findings were observed postoperatively. Cardiac surgery with cardiopulmonary bypass causes systemic inflammation [3, 8]. In addition, patients in our study, generally fasted on the day of surgery and received antibiotics and anesthetic agents perioperatively. Antibiotics cause damage to the gut flora [10,22]. In this study, microbiota counts after cardiac surgery were significantly lower and gut flora components changed.

Postoperative Lactobacillus counts were significantly lower. Lactobacillus are a major component of the gut flora. Many studies have investigated the relationship between Lactobacillus counts and disease [21, 22]. Lower Lactobacillus counts have been observed in irritable bowel syndrome, type 1 diabetes, and multiple sclerosis but higher counts have been observed in colon disease and rheumatoid arthritis. The clinical implications of a lower Lactobacillus count after cardiac surgery are not immediately apparent. Decreases in total microbiota counts may have important clinical implications. The vaginal microbiota is characterized by low diversity and Lactobacillus colonization, which may play an important role in microbiota hemostasis [23].

SCFAs including butyrate, propionate, and acetate play key roles in gut barrier function [24], gut motility, and immune response [25]. SCFAs, which have neuroactive properties, might be directly or indirectly involved in communication along the microbiota-gut-brain axis [26]. SCFAs have been implicated in a range of neuropsychiatric disorders, such as Parkinson disease, Alzheimer disease, and depression [27]. Fecal SCFA concentrations are lower in patients with depression than in controls [28]. Furthermore, butyrate administration was associated with recovery of memory function and increased expression of genes implicated in associative learning in a mouse model of Alzheimer disease [29]. SCFAs may be associated with postoperative mental disorder. A significant decrease in total fecal acid concentrations might be associated with postoperative delirium. Unfortunately, in this study, the number of patients with postoperative delirium may be too small to identify any associations between SCFA concentrations and postoperative delirium. However, postoperative fecal $\mathrm{pH}$ was significantly higher than preoperative $\mathrm{pH}$, which might be associated with postoperative mental disorder. Bacteria which secrete SCFA, such as Lactobacillus decreased postoperatively, suggesting microbiome was damaged and led to leaky gut syndrome and bacterial translocation. Consequently, central nerve system may be influenced.

Antibiotics have a significant impact on the gut microbiota [30]. Oral or intravenous antibiotic use might reduce the gut microbiota. Furthermore, Kohler et al. 
reported that infections and antibiotics are associated with a risk of severe mental disorders [31]. Some reports have claimed that probiotic intake reduces scores on the Depression Anxiety Stress Scale [6]. Antibiotics could modulate components of the gut microbiota possibly, resulting in mental disorders.

Higher Staphylococcus and Pseudomonas counts were observed in patients with pseudopsia in this study. A previous study reported that the peak Staphylococcus and Enterococcus count were associated with the risk of enteritis in patients with systemic inflammatory response syndrome [32]. Pseudomonas is an important pathogen [33]. Pseudomonas in the gut flora increases very quickly in severely ill patients [34]; increased Pseudomonas count is associated with septic complications in patients with systemic inflammatory response syndrome [32]. Oral probiotic intake reduces the incidence of Pseudomonas infections [35]. Damaged gut flora could cause leaky gut syndrome, which is associated with bacterial translocation or bacterial toxins translocation [36]. Lipopolysaccharide (LPS) is a component of gram-negative bacteria such as Pseudomonas. [37] LPS translocation could be occurring in leaky gut syndrome. LPS could evoke strong systemic inflammation $[38,39]$. Increased gram-negative bacteria levels in the gut flora could increase LPS translocation. Some past studies have reported that brain damage is related to gut dysbiosis or endotoxemia [38, 40]. Increased Pseudomonas counts might be related to more severe illness. However, it is not clear from this study whether increased Pseudomonas counts are directly related to postoperative pseudopsia. Further study is necessary to understand the relationship.

There were some limitations to this study. The most obvious limitation in this research was that of a small sample size. Also, before 21 study participants were recruited, 23 potential participants declined to participate because the study design imposes a burden on each participant just after cardiac surgery. Thus, selection bias was likely present. Although Lactobacillus preparations are very popular in Japan, habitual Lactobacillus preparation intake among $47.6 \%$ of patients seems high. Patients with interest in Lactobacillus might have been more likely to participate.

Moreover, there were no patients with delirium among 21 patients after on-pump cardiac surgery, which was not the case in previous studies $[1,41]$. This might be due to severely ill patients not meeting the study eligibility criteria. The assessment of delirium was based on the CAMICU, which is a categorical, not quantitative, diagnosis. It was possible that some cases of hypoactive delirium were not detected. Pseudopsia is considered to be a symptom of delirium that may be associated with the pathophysiology of delirium $[13,42]$.

Even if some limitations existed, this study is valuable in that the gut flora after cardiac surgery was investigated with fecal samples instead of rectal swabs. This is the pivot study to avoid the risk of statistical analysis standard error for small population. This allowed for objective evaluation of microbiota counts and fecal organic acid concentrations. In the field of postoperative cardiac care, induction of symbiotic therapy is considered to be easier after cardiac surgery than after abdominal surgery because the gastrointestinal tract is not damaged directly. Further study is necessary to verify the usefulness of perioperative cardiac symbiotic therapy.

\section{Conclusion}

The gut microbiota can be damaged by cardiac surgical procedures, antibiotics and fasting, and the damage can persist for more than 1 week. Furthermore, Higher Staphylococcus and Pseudomonas counts were observed in patients with pseudopsia.

\section{Abbreviations \\ LPS: Lipopolysaccharide; qRT-PCR: Quantitative reverse transcription polymer- ase chain reaction; POD: Postoperative days; ICU: Intensive care unit; SCFAs: Short-chain fatty acids concentrations.}

\section{Authors' contributions}

All authors have read and approved the manuscript. Indicate authors' role in study concept and design, acquisition of subjects and/or data, analysis and interpretation of data, and preparation of manuscript. MM: Preparation manuscript, KY: Preparation manuscript, MY: Acquisition of data, TA: analysis and interpretation of data, YS: analysis and interpretation of data, SF: study concept and design, NT: Acquisition of data, TF: study concept and design, YO: study concept and design. All authors read and approved the final manuscript.

\section{Funding}

Institutional funding.

\section{Ethics approval and consent to participate}

Institutional Ethic Committee approved this study (M29-053) and written informed consent was obtained in each patient. The Declaration of Helsinki and its subsequent revisions were followed.

\section{Consent for publication}

Not applicable.

\section{Competing interests}

All authors have no conflict of interest.

Sponsor's role

Yakult Central Institute: analysis and preparation of paper.

\section{Author details}

${ }^{1}$ Department of Anesthesiology, National Cerebral and Cardiovascular Center, Suita, Japan. ${ }^{2}$ Yakult Central Institute, Kunitachi, Japan. ${ }^{3}$ Department of Cardiovascular Surgery, National Cerebral and Cardiovascular Center, Suita, Japan.

Received: 15 August 2020 Accepted: 12 October 2020

Published online: 20 October 2020

References

1. Jarvela K, Porkkala H, Karlsson S, Martikainen T, Selander T, Bendel S. Postoperative delirium in cardiac surgery patients. J Cardiothorac Vasc Anesth. 2018;32(4):1597-602. 
2. Gernhardt C, Kluge S, Meon M, Schmidtke C, Karluss A, Sedemund-Adib B, Wetterling T, Sievers $\mathrm{HH}$, Junghanns K. Risk factors for postoperative delirium after cardiac surgery. Fortschr Neurol Psychiatr. 2017;85(5):274-9.

3. Evora PR, Bottura C, Arcencio L, Albuquerque AA, Evora PM, Rodrigues AJ. Key points for curbing cardiopulmonary bypass inflammation. Acta Cir Bras. 2016;31 (Suppl 1):45-52.

4. Cresci GA, Bawden E. Gut microbiome: what we do and don't know. Nutr Clin Pract. 2015;30(6):734-46.

5. Jandhyala SM, Talukdar R, Subramanyam C, Vuyyuru H, Sasikala M, Nageshwar Reddy D. Role of the normal gut microbiota. World I Gastroenterol. 2015;21(29):8787-803.

6. Fung TC, Olson CA, Hsiao EY. Interactions between the microbiota, immune and nervous systems in health and disease. Nat Neurosci. 2017;20(2):145-55.

7. Quigley EMM. Microbiota-brain-gut axis and neurodegenerative diseases. Curr Neurol Neurosci Rep. 2017;17(12):94.

8. Belhaj A. Actual knowledge of systemic inflammation reaction during cardiopulmonary bypass. Recent Pat Cardiovasc Drug Discov. 2012;7(3):165-9.

9. Maier L, Pruteanu M, Kuhn M, Zeller G, Telzerow A, Anderson EE, Brochado $A R$, Fernandez KC, Dose $\mathrm{H}$, Mori $\mathrm{H}$, et al. Extensive impact of non-antibiotic drugs on human gut bacteria. Nature. 2018;555(7698):623-8.

10. Shimizu K, Ogura H, Goto M, Asahara T, Nomoto K, Morotomi M, Yoshiya K, Matsushima A, Sumi Y, Kuwagata Y, et al. Altered gut flora and environment in patients with severe SIRS. J Trauma. 2006;60(1):126-33.

11. Lederer AK, Pisarski P, Kousoulas L, Fichtner-Feigl S, Hess C, Huber R. Postoperative changes of the microbiome: are surgical complications related to the gut flora? A systematic review. BMC Surg. 2017;17(1):125

12. Gusmao-Flores D, Salluh Jl, Chalhub RA, Quarantini LC. The confusion assessment method for the intensive care unit (CAM-ICU) and intensive care delirium screening checklist (ICDSC) for the diagnosis of delirium: a systematic review and meta-analysis of clinical studies. Crit Care. 2012;16(4):R115.

13. Sockalingam S, Parekh N, Bogoch II, Sun J, Mahtani R, Beach C, Bollegalla $\mathrm{N}$, Turzanski S, Seto E, Kim J, et al. Delirium in the postoperative cardiac patient: a review. J Card Surg. 2005;20(6):560-7.

14. Matsuda K, Tsuji H, Asahara T, Kado Y, Nomoto K. Sensitive quantitative detection of commensal bacteria by rRNA-targeted reverse transcriptionPCR. Appl Environ Microbiol. 2007;73(1):32-9.

15. Matsuda K, Tsuji H, Asahara T, Matsumoto K, Takada T, Nomoto K. Establishment of an analytical system for the human fecal microbiota, based on reverse transcription-quantitative PCR targeting of multicopy rRNA molecules. Appl Environ Microbiol. 2009;75(7):1961-9.

16. Sakaguchi S, Saito M, Tsuji H, Asahara T, Takata O, Fujimura J, Nagata S, Nomoto K, Shimizu T. Bacterial rRNA-targeted reverse transcription-PCR used to identify pathogens responsible for fever with neutropenia. J Clin Microbiol. 2010;48(5):1624-8.

17. Nishigaki E, Abe T, Yokoyama Y, Fukaya M, Asahara T, Nomoto K, Nagino $M$. The detection of intraoperative bacterial translocation in the mesenteric lymph nodes is useful in predicting patients at high risk for postoperative infectious complications after esophagectomy. Ann Surg. 2014;259(3):477-84

18. Matsuda K, Tsuji H, Asahara T, Takahashi T, Kubota H, Nagata S, Yamashiro Y, Nomoto K. Sensitive quantification of Clostridium difficile cells by reverse transcription-quantitative PCR targeting rRNA molecules. Appl Environ Microbiol. 2012;78(15):5111-8.

19. Tsuji H, Matsuda K, Nomoto K. Counting the countless: bacterial quantification by targeting rRNA molecules to explore the human gut microbiota in health and disease. Front Microbiol. 2018;9:1417.

20. Yokoyama Y, Mizuno T, Sugawara G, Asahara T, Nomoto K, Igami T, Ebata T, Nagino M. Profile of preoperative fecal organic acids closely predicts the incidence of postoperative infectious complications after major hepatectomy with extrahepatic bile duct resection: importance of fecal acetic acid plus butyric acid minus lactic acid gap. Surgery. 2017;162(4):928-36.

21. Heeney DD, Gareau MG, Marco ML. Intestinal Lactobacillus in health and disease, a driver or just along for the ride? Curr Opin Biotechnol. 2018:49:140-7.

22. O'Callaghan J, O'Toole PW. Lactobacillus: host-microbe relationships. Curr Top Microbiol Immunol. 2013;358:119-54.

23. Collins SL, McMillan A, Seney S, van der Veer C, Kort R, Sumarah MW, Reid G. Promising prebiotic candidate established by evaluation of lactitol, lactulose, raffinose, and oligofructose for maintenance of a lactobacillus-dominated vaginal microbiota. Appl Environ Microbiol. 2018:84(5):e02200-17.

24. Matsumoto M, Inoue R, Tsukahara T, Ushida K, Chiji H, Matsubara N, Hara H. Voluntary running exercise alters microbiota composition and increases $n$-butyrate concentration in the rat cecum. Biosci Biotechnol Biochem. 2008:72(2):572-6.

25. De Filippo C, Cavalieri D, Di Paola M, Ramazzotti M, Poullet JB, Massart S, Collini S, Pieraccini G, Lionetti P. Impact of diet in shaping gut microbiota revealed by a comparative study in children from Europe and rural Africa. Proc Natl Acad Sci USA. 2010;107(33):14691-6.

26. Stilling RM, van de Wouw M, Clarke G, Stanton C, Dinan TG, Cryan JF. The neuropharmacology of butyrate: The bread and butter of the microbiotagut-brain axis? Neurochem Int. 2016;99:110-32.

27. Kalantar-Zadeh K, Berean KJ, Burgell RE, Muir JG, Gibson PR. Intestinal gases: influence on gut disorders and the role of dietary manipulations. Nat Rev Gastroenterol Hepatol. 2019;16(12):733-47.

28. Szczesniak O, Hestad KA, Hanssen JF, Rudi K. Isovaleric acid in stool correlates with human depression. Nutr Neurosci. 2016;19(7):279-83.

29. Bedarf JR, Hildebrand F, Coelho LP, Sunagawa S, Bahram M, Goeser F, Bork $\mathrm{P}$, Wullner U. Functional implications of microbial and viral gut metagenome changes in early stage L-DOPA-naive Parkinson's disease patients. Genome Med. 2017:9(1):39.

30. Iizumi T, Battaglia T, Ruiz V, Perez Perez Gl. Gut microbiome and antibiotics. Arch Med Res. 2017;48(8):727-34.

31. Kohler O, Petersen L, Mors O, Mortensen PB, Yolken RH, Gasse C, Benros $M E$. Infections and exposure to anti-infective agents and the risk of severe mental disorders: a nationwide study. Acta Psychiatr Scand. 2017;135(2):97-105.

32. Shimizu K, Ogura H, Hamasaki T, Goto M, Tasaki O, Asahara T, Nomoto K, Morotomi M, Matsushima A, Kuwagata Y, et al. Altered gut flora are associated with septic complications and death in critically ill patients with systemic inflammatory response syndrome. Dig Dis Sci. 2011;56(4):1171-7.

33. Bodey GP, Bolivar R, Fainstein V, Jadeja L. Infections caused by Pseudomonas aeruginosa. Rev Infect Dis. 1983;5(2):279-313.

34. Hayakawa M, Asahara T, Henzan N, Murakami H, Yamamoto H, Mukai $N$, Minami Y, Sugano M, Kubota N, Uegaki S, et al. Dramatic changes of the gut flora immediately after severe and sudden insults. Dig Dis Sci. 2011;56(8):2361-5.

35. Forestier C, Guelon D, Cluytens V, Gillart T, Sirot J, De Champs C. Oral probiotic and prevention of Pseudomonas aeruginosa infections: a randomized, double-blind, placebo-controlled pilot study in intensive care unit patients. Crit Care. 2008;12(3):R69.

36. Obrenovich MEM. Leaky gut, leaky brain? Microorganisms. 2018;6(4):107.

37. King JD, Kocincova D, Westman EL, Lam JS. Review: lipopolysaccharide biosynthesis in Pseudomonas aeruginosa. Innate Immun. 2009;15(5):261-312.

38. Yamashiro K, Tanaka R, Urabe T, Ueno Y, Yamashiro Y, Nomoto K, Takahashi T, Tsuji H, Asahara T, Hattori N. Gut dysbiosis is associated with metabolism and systemic inflammation in patients with ischemic stroke. PLoS ONE. 2017;12(2):e0171521.

39. Tamaki S, Kanazawa A, Sato J, Tamura Y, Asahara T, Takahashi T, Matsumoto S, Yamashiro Y, Watada H. Clinical factors associated with bacterial translocation in Japanese patients with type 2 diabetes: a retrospective study. PLoS ONE. 2019;14(9):e0222598.

40. Kurita N, Yamashiro K, Kuroki T, Tanaka R, Urabe T, Ueno Y, Miyamoto N, Takanashi M, Shimura H, Inaba T, et al. Metabolic endotoxemia promotes neuroinflammation after focal cerebral ischemia. J Cereb Blood Flow Metab. 2020.

41. Rengel KF, Pandharipande PP, Hughes CG. Postoperative delirium. Presse Med. 2018;47(4 Pt 2):e53-64.

42. Bergeron N, Dubois MJ, Dumont M, Dial S, Skrobik Y. Intensive Care Delirium Screening Checklist: evaluation of a new screening tool. Intensive Care Med. 2001;27(5):859-64.

\section{Publisher's Note}

Springer Nature remains neutral with regard to jurisdictional claims in published maps and institutional affiliations. 